\title{
Lattice study of the scalar and baryon spectra in many-flavor QCD
}

\author{
Yasumichi Aoki ${ }^{a}$, Tatsumi Aoyama ${ }^{a}$, Ed Bennett ${ }^{b}$, Masafumi Kurachi $^{c}$, Toshihide Maskawa $^{a}$, \\ Kohtaroh Miura $^{d}$, Kei-ichi Nagai ${ }^{a}$, Hiroshi Ohki ${ }^{e}$, Enrico Rinaldi ${ }^{f}$, Akihiro Shibata ${ }^{g}$, Koichi \\ Yamawaki $^{a}$, and Takeshi Yamazaki ${ }^{h}$ \\ (LatKMI Collaboration) \\ a Kobayashi-Maskawa Institute for the Origin of Particles and the Universe (KMI), Nagoya \\ University, Nagoya, 464-8602, Japan \\ ${ }^{b}$ Department of Physics, Swansea University, Singleton Park, Swansea SA2 8PP, UK \\ ${ }^{c}$ Institute of Particle and Nuclear studies, High Energy Accelerator Research Organization \\ (KEK), Tsukuba 305-0801, Japan \\ d Centre de Physique Theorique(CPT), Aix-Marseille Univerisity, Campus de Luminy, Case \\ 907, 163 Avenue de Luminy, 13288 Marseille cedex 9, France \\ e RIKEN/BNL Research center, Brookhaven National Laboratory, Upton, NY, 11973, USA \\ $f$ Lawrence Livermore National Laboratory, Livermore, California, 94550, USA \\ $g$ Computing Research Center, High Energy Accelerator Research Organization (KEK), Tsukuba \\ 305-0801, Japan \\ $h$ Graduate School of Pure and Applied Sciences, University of Tsukuba, Tsukuba, Ibaraki \\ 305-8571, Japan
}

\begin{abstract}
In the search for a composite Higgs boson in walking technicolor models, many flavor QCD, in particular with $N_{f}=8$, is an attractive candidate, and has been found to have a composite flavor-singlet scalar as light as the pion. Based on lattice simulations of this theory with the HISQ action, we will present our preliminary results on the scalar decay constant using the fermionic bilinear operator, and on the mass of the lightest baryon state which could be a dark matter candidate. Combining these two results, implications for dark matter direct detection are also discussed.
\end{abstract}

Keywords: Lattice gauge theory, Conformal/Walking Dynamics, Dark matter, composite Higgs particle.

\section{Introduction}

A new scalar particle with mass $125 \mathrm{GeV}$ has been discovered at the LHC, and the current experimental data is consistent with the standard model (SM) of particle physics. However, the origin of mass and electroweak symmetry breaking remains unknown, and the SM does not have a viable dark matter (DM) candidate, which are reasons why we are seeking new physics beyond the SM.

It is possible that there is strong gauge dynamics beyond the $\mathrm{TeV}$ scale: one popular candidate is the Walking Technicolor model. This breaks the electroweak symmetry dynamically in an approximately scale-invariant dynamics, and predicts a large anomalous dimension $\gamma_{m} \simeq 1$ together with a "techni-dilaton" as a pseudo Nambu-Goldstone boson of the spontaneously broken approximate scale symmetry $\stackrel{\underline{1}}{\underline{1}}$. The Higgs scalar could be identified with the techni-dilaton, which is a 
composite bound-state of the flavor-singlet techni-fermion bilinear, parametrically lighter than other techni-hadrons. From a phenomenological point of view, the techni-dilaton decay constant (denoted here as $F_{\sigma}$ ) is a very important parameter in addition to its mass, since $F_{\sigma}$ controls all the techni-dilaton's couplings to SM particles. In addition, the technicolor model has a rich hadron structure, and composite resonances like the techni-rho meson $(\rho)$ may be in the discovery reach of run-II at the LHC. Furthermore the techni-baryon, its lightest neutral component, could be a stable particle due to techni-fermion number conservation. Thus it would be a candidate for DM.

In order to study the walking technicolor model, non-perturbative understanding of the strong gauge dynamics is necessary, and lattice numerical calculations are the most powerful tool for that purpose. In a previous lattice study, the LatKMI collaboration has shown that 8-flavor $\left(N_{f}=8\right)$ QCD could be a candidate for a walking gauge theory. We found an approximate hyperscaling relation for various hadron masses in a certain fermion mass range $\underset{2}{2}$. Remarkably, we also found that the flavor singlet-scalar $(\sigma)$ mass is as light as the pseudoscalar $(\pi)$, which might indicate that $\sigma$ could be regarded as the techni-dilaton ${ }^{3}$.

In this proceedings we investigate the decay constant of the flavor-singlet scalar ant its mass. Using the Ward-Takahashi (WT) relation of the scale symmetry in the continuum theory, we estimate the dilaton decay constant $F_{\sigma}$. As for the techni-baryon DM, it is important to investigate its direct detection, where the most dominant contribution of the DM scattering amplitude is the scalar mediated interaction. Based on a DM effective theory whose parameters include $F_{\sigma}$ and the techni-baryon mass, we evaluate the leading order of the scattering cross section of the DM. Using the lattice input for the above parameters, we discuss experimental detectability and the possible realization of the techni-baryon DM scenario.

In the next section, we briefly explain how to calculate the mass of the flavorsinglet scalar and scalar decay constant on the lattice. We discuss a relation between the scalar and dilaton decay constants. In Section 3, we show our lattice result of the scalar mass and decay constants, including a chiral extrapolation based on a dilaton effective theory. In Section 4, we study the DM detection rate based on the dilaton chiral perturbation theory with baryon, and the summary is given in Section 5. Note that all the results shown in this proceeding are preliminary.

\section{Scalar mass and decay constant}

We investigate the mass of the scalar and its decay constant on the lattice. The mass of the flavor-singlet scalar, which we call $\sigma$, is calculated from the two-point correlation function $\left(C_{\sigma}(t)\right)$ of the flavor-singlet scalar bilinear operator $\mathcal{O}_{s}$. By using the staggered lattice fermion field $\chi_{i}, \mathcal{O}_{s}$ is written as $\mathcal{O}_{s}(x, t)=\sum_{i} \bar{\chi}_{i}(x, t) \chi_{i}(x, t)$, where $i$ denotes the different staggered fermion species, namely $i=1,2$ for $N_{f}=8$ QCD. The correlator is then given by

$$
C_{\sigma}(t)=\frac{1}{V} \sum_{x}\left\langle\mathcal{O}_{s}(x, t) \mathcal{O}_{s}(0,0)\right\rangle,
$$


where $V$ is the spatial volume $\left(V=L^{3}\right)$. The asymptotic behavior of $C_{\sigma}(t)$ is given by $C_{\sigma}(t)=A_{\sigma}(t)+(-1)^{t} A_{\pi \overline{S C}}(t)$, where $A_{\pi_{\overline{S C}}}$ is a pseudoscalar correlator, and $A_{H}(t)=A_{H}\left(e^{-m_{H} t}+e^{-m_{H}(T-t)}\right)$ for different hadrons $H$.

We define the scalar decay constant $F_{S}$ as the scalar operator matrix element,

$$
\left\langle 0\left|m_{f} O_{s}(0,0)\right| \sigma(0)\right\rangle=F_{S} m_{\sigma}^{2} .
$$

The above matrix element can be calculated from $C_{\sigma}(t)$. Substituting the complete set, $\sum_{n}|n\rangle\langle n|=\int \frac{d^{3} p}{(2 \pi)^{3}} \frac{|\sigma(p)\rangle\langle\sigma(p)|}{2 E_{p}}+\cdots$ into Eq. 1, the correlator $C_{\sigma}(t)$ is given by

$$
C_{\sigma}(t)=\frac{1}{V}\left|\left\langle 0\left|O_{s}(0,0)\right| \sigma(0)\right\rangle\right|^{2} \frac{1}{2 m_{\sigma}}\left(e^{-m_{\sigma} t}+e^{-m_{\sigma}(T-t)}\right) .
$$

Thus we calculate $F_{S}$ as follows,

$$
F_{S}=\frac{m_{f} \sqrt{2 m_{\sigma} V A_{\sigma}}}{m_{\sigma}^{2}} .
$$

We note that this quantity is renormalization group invariant and a physical quantity, and it is easy to measure on the lattice. It is also noted that $F_{S}$ should obey a hyperscaling relation, $F_{S} \propto m_{f}^{1 /(1+\gamma)}$ with $\gamma \sim \gamma_{m}$, if the theory is in the conformal window ${ }^{4}$.

Let us now discuss the dilaton decay constant $F_{\sigma}$. The following discussion is based on the continuum theory. It is important to recall the basic definition of the dilaton decay constant, which is $\left\langle 0\left|\mathcal{D}^{\mu}(x)\right| \sigma(p)\right\rangle=-i F_{\sigma} p^{\mu} e^{-i p x}$. From this we obtain $\left\langle 0\left|\partial_{\mu} \mathcal{D}^{\mu}(0)\right| \sigma(0)\right\rangle=-F_{\sigma} m_{\sigma}^{2}$. Therefore the dilaton decay constant can be directly calculated from the matrix element of the dilatation current. However the dilatation current is rather difficult to construct on the lattice, since it contains a power divergence that needs to be subtracted.

Instead, we consider an alternative way to estimate it, which can be derived by using the WT relation for the dilatation current. Following the argument in 5 , we consider the integrated WT relation for dilatation transformation. We use the scale transformation relation of an operator $\mathcal{O}, \delta_{D} \mathcal{O}=\Delta_{\mathcal{O}} \mathcal{O}$, where $\Delta_{\mathcal{O}}$ is the scaling dimension of $\mathcal{O}$. In the zero momentum transfer limit the WT relation leads to $\int d^{4} x T\left\langle\partial_{\mu} \mathcal{D}^{\mu}(x) \mathcal{O}(0)\right\rangle=\Delta_{\mathcal{O}}\langle\mathcal{O}\rangle$ a, from which, by assuming the $\sigma$ pole dominance, we obtain $\Delta_{\mathcal{O}}\langle\mathcal{O}\rangle=\left(-F_{\sigma} m_{\sigma}^{2}\right) \frac{1}{m_{\sigma}^{2}}\langle\sigma|\mathcal{O}| 0\rangle=-F_{\sigma}\langle\sigma|\mathcal{O}| 0\rangle \mathrm{b}$ In the case we take $\mathcal{O}(x)=m_{f} \sum_{i}^{N_{F}} \bar{\psi}_{i} \psi_{i}(x)$ (the flavor-singlet scalar operator), we obtain a relation,

$$
F_{S} F_{\sigma} m_{\sigma}^{2}=-\Delta_{\bar{\psi} \psi} m_{f} \sum_{i}^{N_{F}}\left\langle\bar{\psi}_{i} \psi_{i}\right\rangle .
$$

(Dividing both sides by $m_{f}$ leads to the relation obtained at $m_{f}=0 \underline{\underline{6}}$.)

We note that this relation holds in the continuum theory with infrared conformality under the assumption of the $\sigma$ pole dominance. While we do not know the

\footnotetext{
a In the formula, the vacuum contribution on the left hand side is absent in the WT relation.

b The so-called Partially Conserved Dilatation Current (PCDC) relation $F_{\sigma}^{2} m_{\sigma}^{2}=-4\left\langle\theta_{\mu}^{\mu}\right\rangle$ follows for $\mathcal{O}=\partial_{\mu} \mathcal{D}^{\mu}=\theta_{\mu}^{\mu}$ (nonperturbative trace anomaly).
} 
real infrared behavior in $N_{f}=8 \mathrm{QCD}$ towards the chiral limit, our recent lattice study shows that an approximate hyperscaling relation (walking behavior) is found for various hadron masses in a certain fermion mass region of $m_{f}$, so the above relation could be effective also in the walking region up to excited state contaminations and discretization effects. Therefore in the following preliminary lattice analysis, we shall use this relation for a semi-direct estimate of $F_{\sigma}$. In order to precisely calculate $F_{\sigma}$, however, a direct calculation of the dilatation current is needed.

\section{Result}

In this section we show our lattice results for $N_{f}=8 \mathrm{QCD}$. Details of the simulation and analysis for measurements can be found in $\stackrel{8}{ }$. The results for $m_{\sigma}$ and $F_{S}$ are summarized in Fig 1 It is found that the scalar is as light as $\pi$, and clearly lighter than $\rho$, and we obtain a good signal for $F_{S}$ as well. The lightness of the scalar could be regarded as a reflection of a dilatonic nature of the scalar.

While our fermion mass is far from the chiral limit, we shall study the chiral extrapolation based on an effective theory, which is dilaton chiral perturbation theory $(\mathrm{DChPT})^{\underline{7}}$. At the leading order of the DChPT, the scalar mass is given by $m_{\sigma}^{2}=d_{0}+d_{1} m_{\pi}^{2}$, where $d_{1}=\frac{\left(3-\gamma_{m}\right)\left(1+\gamma_{m}\right)}{4} \frac{N_{f} F_{\pi}^{2}}{F_{\sigma}^{2}}, m_{\pi}$ and $F_{\pi}$ are the mass and decay constant of $\pi$. We also try a fit with a naive form of $m_{\sigma}=c_{0}+c_{1} m_{f}$. The fit results are shown in the left panel of Fig. 2 where a reasonable value of $\chi^{2} /$ dof $\sim \mathcal{O}(1)$ is obtained. The value of $m_{\sigma}^{2}$ in the chiral limit is $d_{0}=-0.0028(98)$. Thus we obtain a very light $\sigma$. From the fit value of the slope $d_{1}=0.89(26)$ in DChPT, we also obtain $F_{\sigma} \sim \sqrt{N_{f}} F_{\pi} \sim 0.06$, with $F_{\pi}$ being in the chiral limit $\underline{\underline{8}}$ and $\gamma \sim \gamma_{m} \sim 1$.

On the right panel of Fig. 2 the result of $F_{\sigma}$ from the semi-direct estimate (Eq. 5) is shownd. We also carry out the chiral extrapolation fits, whose results are also shown in the figure. In the chiral limit, we obtain $\frac{F_{\sigma}}{\Delta_{\bar{\psi}} w} \sim 0.03$. Given that $\Delta_{\bar{\psi} \psi}=3-\gamma$, we have shown how two different methods, DChPT and a semi-direct calculation, give a consistent result for $F_{\sigma}$.
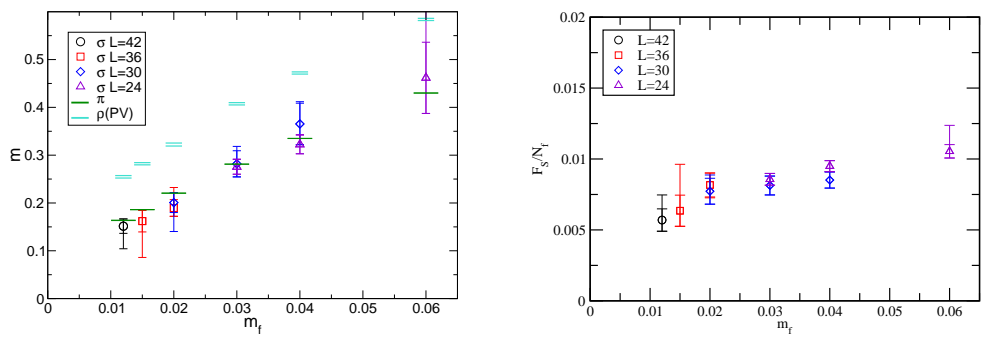

Fig. 1. (Left) Mass of the flavor-singlet scalar. Other hadron masses are also shown. Outer error represents the statistical and systematic uncertainties added in quadrature, while inner error is only statistical. (Right) Scalar decay constant of the flavor-singlet scalar.

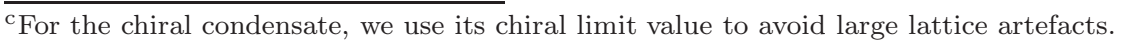



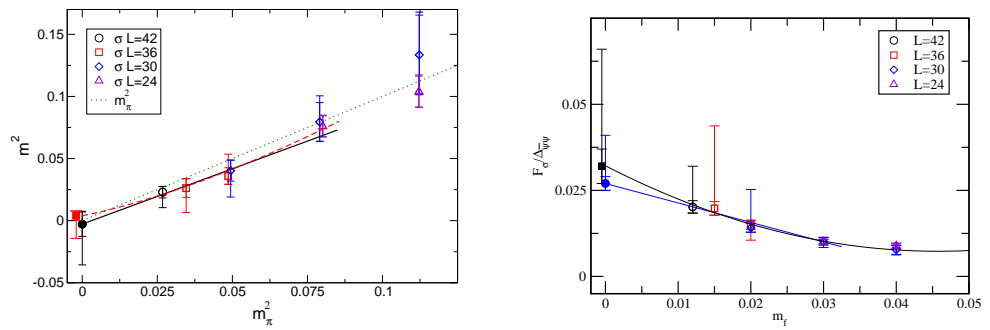

Fig. 2. (Left) $m_{\sigma}^{2}$ v.s. $m_{\pi}^{2}$. Black line shows the fit with DChPT formula. Dashed red curve shows the fit result with a naive form, $m_{\sigma}=c_{0}+c_{1} m_{f}$. (Right) Dilaton decay constant from a semidirect method. Blue and Black lines show the chiral fits of the linear and quadratic polynomial in $m_{f}$ with the lightest 4 and 5 data points used. Outer error represents the statistical and systematic uncertainties added in quadrature, while inner error is only statistical.

\section{Dilaton ChPT with baryon and dark matter}

The technicolor model may provide a good candidate for composite DM, in the form of a neutral baryonic bound state made of constituent (possibly charged) technifermions. In this case, the coupling between the SM particle and the DM as well as the mass of the DM are constrained by direct detection experiments. In most cases, the experiments determine the scattering rate of DM with heavy nuclei in the detector, one dominant contribution to which is the Higgs (scalar) mediated spinindependent process. In the following, we provide a low-energy effective theory of the walking gauge theory including the DM, whose low-energy constants can be determined from the lattice results of the baryon and scalar spectra. The result obtained in the previous section is useful, and it gives rise to information for the DM direct detection experiments.

We consider a DM effective theory including the dilaton based on the DChPT. Since the DM is the lightest techni-baryon, the extension to the baryon sector of the DChPT is straightforward $\stackrel{7}{7}$. As a result, in the leading order the dilaton field can only couple through the baryon mass term as

$$
\mathcal{L}=\bar{B}(x)\left(i \gamma_{\mu} \partial^{\mu}-\chi(x) m_{B}\right) B(x),
$$

where $\chi(x)=e^{\sigma(x) / F_{\sigma}}, B(x)$ is the baryon field, and $m_{B}$ is its mass in the chiral limit. The parameter $m_{B}$ explicity breaks the scale symmetry, and the (pseudo) dilaton acts on this term to make the action scale invariant. Actually this action is invariant under the scale transformation, $\delta B=\left(\frac{3}{2}+x_{\nu} \partial^{\nu}\right) B, \delta \chi=\left(1+x_{\nu} \partial^{\nu}\right) \chi$.

From this effective theory, we can read off the dilaton-baryon effective coupling $\left(y_{\bar{B} B \sigma}\right)$, which is uniquely determined as $y_{\bar{B} B \sigma}=m_{B} / F_{\sigma}$. Regarding to the SM sector, the dilaton-nucleon coupling is also determined within the framework of the dilaton effective theory $\underline{\underline{10}}$, since the dilaton-quark coupling $\left(y_{\sigma \bar{f} f}\right)$ could be related to the SM Yukawa coupling $\left(y_{h_{\mathrm{SM}} \bar{f} f}\right)$ as $\frac{y_{\sigma \bar{f} f}}{y_{h_{\mathrm{SM}} \bar{f} f}}=\frac{(3-\gamma) v_{E W}}{F_{\sigma}}$. Combining both $\mathrm{SM}$ and technicolor sectors, the cross section with a target nucleus $N$ is given as $\sigma_{S I}=\frac{M_{R}^{2}}{\pi}\left(Z f_{p}+(A-Z) f_{n}\right)^{2}$, where $M_{R}=\left(m_{B} m_{N}\right) /\left(m_{B}+m_{N}\right)$, and $Z$ and 
$A$ are the total number of the protons $(p)$ and neutrons $(n)$ in the nucleus. The parameter $f_{(n, p)}$ is defined as $f_{(n, p)}=\frac{m_{N}}{\sqrt{2} m^{2}} \frac{y_{\bar{B} B \sigma}}{F_{\sigma}}(3-\gamma)\left(\sum_{q=u, d, s} f_{T_{q}}^{(n, p)}+\frac{2}{9} f_{T_{G}}^{(n, p)}\right)$, where $f_{T_{q}}^{(n, p)}$ is the nucleon $\sigma$-term of the light quarks $(q=u, d, s)$, and $f_{T_{G}}^{(n, p)}$ is that of the heavy quark. d. Thus lattice calculations are used in the technicolor theory as well as in QCD theory to obtain non-perturbative information about DM physics.

Here we show our numerical results of the DM cross sectione. We use the lattice results of the dilaton decay constant $\left(F_{\sigma}\right)$ obtained from the previous section and baryon mass, while the scalar mass $m_{\sigma}$ is fixed to its experimental value $(125 \mathrm{GeV})$ in this analysis. We use the values in ${ }^{9}$ for $f_{T_{q}}^{(n, p)}$. To set the scale, we use the relation $\sqrt{N_{f} / 2} F_{\pi} / \sqrt{2}=246 \mathrm{GeV}$. We again use the $F_{\pi}$ in the chiral limit. To compare it with experiment, we use the cross section per nucleon $\left(\sigma_{0}\right)$ instead of $\sigma_{S I}$. The result is shown in Fig. 3. According to DM direct detection experiment:f, our values for $\sigma_{0}$ are excluded, so that it may be difficult to explain the existence of DM as a techni-baryon. However we note that there exist other contributions to the DM cross section, e.g. gauge boson mediated interaction, and higher order terms, which might affect the DM cross section. Calculation of these contributions on the lattice is left for future investigations.

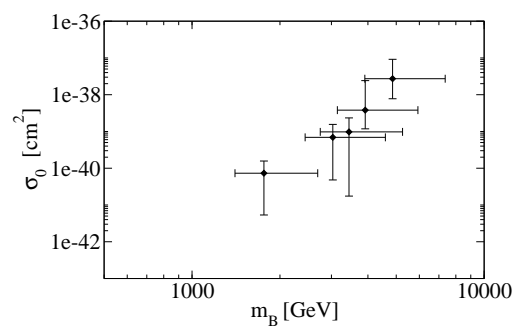

Fig. 3. $\quad \sigma_{0}\left[\mathrm{~cm}^{2}\right]$ as a function of $m_{B}[\mathrm{GeV}]$. The results for $m_{f}=0.030,0.020,0.015,0.012$, and the chiral limit from upper-right to lower-left. Both the statistical and systematic errors are included. Experimentally allowed region is below the plotted window.

\section{Summary}

The scalar mass and decay constant are very important parameters to probe a technicolor signature at the LHC. Based on the lattice theory, we derived a relation between the scalar decay constant and the (flavor-singlet) scalar correlation functions. Our numerical result shows that the signal of the decay constant is as good as that of the mass. Although the accuracy of our result is not enough to precisely extrapolate towards the chiral limit, we obtained a rough estimate of the

\footnotetext{
$\mathrm{d}$ In general, techni-fermions can be charged under the SM color, so there may exist additional contributions to the nucleon matrix elements from the techni-fermions. In this analysis, we omit these contributions for simplicity.

e A similar analysis on the lattice has been performed for a different composite DM model based on strong dynamics 11 .

${ }^{\mathrm{f}}$ For a recent experiment, see e.g. 12 .
} 
ratio $F_{\sigma} / F_{\pi} \sim 3$. This is useful for collider phenomenology. Besides it, $F_{\sigma}$ can be also used for DM physics. We provided a DM effective theory based on the dilaton ChPT, where a dilaton-DM coupling is related to $F_{\sigma}$ and the DM mass. This coupling is constrained by DM direct detection experiments, e.g. LUX. We then discussed a possible scenario for techni-baryon DM. We note that all the results shown here are preliminary. Simulation at lighter fermion mass, and the direct computations on the lattice are needed to precisely estimate the above quantities.

Acknowledgments - Numerical computations have been carried out on the highperformance computing systems at KMI $(\varphi)$, at the Information Technology Center in Nagoya University (CX400), and at the Research Institute for Information Technology in Kyushu University (CX400 and HA8000). This work is supported by the JSPS Grant-in-Aid for Scientific Research (S) No.22224003, (C) No.23540300 (K.Y.), for Young Scientists (B) No.25800139 (H.O.) and No.25800138 (T.Y.), and also by the MEXT Grants-in-Aid for Scientific Research on Innovative Areas No.23105708 (T.Y.) and No.25105011 (M.K.). This work is supported by the JLDG constructed over the SINET of NII. The work of H.O. is supported by the RIKEN Special Postdoctoral Researcher program. E.R. acknowledges the support of the U.S. Department of Energy under Contract DE-AC52- 07NA27344 (LLNL).

\section{References}

1. K. Yamawaki, M. Bando and K. i. Matumoto, Phys. Rev. Lett. 56, 1335 (1986).

2. Y. Aoki et al. [LatKMI Collaboration], Phys. Rev. D 87, no. 9, 094511 (2013) arXiv:1302.6859 [hep-lat]].

3. Y. Aoki et al. [LatKMI Collaboration], Phys. Rev. D 89, 111502 (2014) arXiv:1403.5000 [hep-lat]].

4. L. Del Debbio and R. Zwicky, Phys. Rev. D 82, 014502 (2010) arXiv:1005.2371 [hep-ph]].

5. M. Bando, K. i. Matumoto and K. Yamawaki, Phys. Lett. B 178, 308 (1986).

6. S. Matsuzaki and K. Yamawaki, Phys. Rev. D 86, 115004 (2012) arXiv:1209.2017 [hep-ph]].

7. S. Matsuzaki and K. Yamawaki, Phys. Rev. Lett. 113, no. 8, 082002 (2014) arXiv:1311.3784 [hep-lat]].

8. LatKMI collaboration in preparation.

9. J. Hisano, K. Ishiwata and N. Nagata, JHEP 1506, 097 (2015) arXiv:1504.00915 [hep-ph]].

10. S. Matsuzaki and K. Yamawaki, Phys. Rev. D 86, 035025 (2012) arXiv:1206.6703 [hep-ph]].

11. T. Appelquist et al. [Lattice Strong Dynamics (LSD) Collaboration], Phys. Rev. D 89, no. 9, 094508 (2014) arXiv:1402.6656 [hep-lat]].

12. D. S. Akerib et al. [LUX Collaboration], Phys. Rev. Lett. 112, 091303 (2014) arXiv:1310.8214 [astro-ph.CO]]. 